\title{
An Ozone Micro-bubble Technique for Seed Sterilization in Alfalfa Sprouts
}

\author{
Yurina Kwack ${ }^{1}$, Kyoung Koo Kim² ${ }^{2}$ Hyunseung Hwang ${ }^{2}$, and Changhoo Chun ${ }^{1,2^{*}}$ \\ ${ }^{1}$ Research Institute of Agriculture and Life Sciences, Seoul National University, Seoul 151-921, Korea \\ ${ }^{2}$ Department of Plant Science, Seoul National University, Seoul 151-921, Korea
}

\begin{abstract}
The efficacy of ozone micro-bubble water (OMBW) in reducing microbial populations on alfalfa seeds was investigated in this study. We observed the surface of alfalfa seeds using microscopy and found that many cracks and crevices existing on the surface could harbor pathogens. Alfalfa seeds were treated with tap water (TW), micro-bubble water (MBW), ozone water (OW), ozone micro-bubble water (OMBW), and chlorine water (CL) for $5 \mathrm{~min}$, and total microbial population, E. coli and Salmonella spp. colonies were determined. Also, the sterilized seeds were germinated and cultivated for $5 \mathrm{~d}$ after sowing to investigate the percentage of germination and the growth of alfalfa sprouts. The treatments with OMBW and CL were most effective in reducing total microbial populations and E. coli was eliminated by OW, OMBW, and CL treatments. CL treatment reduced the percentage of germination and fresh weight of alfalfa sprouts, but OMBW did not cause any negative effects on the germination and growth of alfalfa sprouts. These results indicate that OMBW can be used as an effective sanitizer for eliminating seed-borne pathogens without detrimental effects on seed viability.
\end{abstract}

Additional key words: chlorine, germination, pathogen, sanitizer

\section{Introduction}

In recent years, the popularity of sprouts has increased considerably due to changes in food production and eating habits. Sprouts are used as ingredients of vegetable salads and thus, are often eaten raw. However, outbreaks of human infections associated with the consumption of raw sprouts have also increased. In the late 1990's, there were large outbreaks of food-borne illnesses caused by Escherichia coli and Salmonella spp. due to consumption of fresh vegetable sprouts in Japan and the U.S. (Michino et al., 1999; NACMCF, 1999). Sprout-borne disease outbreaks have also been reported in the U.K., Finland, Denmark, Sweden, and Canada and have involved alfalfa, cress, radish, and mungbean sprouts (Buck et al., 2003).

Seeds have been implicated as the initial inoculum source in sprout-borne disease outbreaks, though practices and conditions in sprout production may increase or decrease the extent of the microbial hazards (NACMCF, 1999; Sharma and Demirci, 2003). Therefore, seed sterilization by physical or chemical treatment is important for reducing the risks of sprout-borne disease outbreaks (Buck et al., 2003).

Physical treatments with heat or ionizing irradiation were explored for seed sterilization. Heat treatment includes exposing seeds to high temperatures for a short period because elevated temperatures kill seed-borne pathogens (Scouten and Beuchat, 2002). Ionizing irradiation has also been shown to reduce seed-borne pathogens because radiation energy can penetrate seed tissues and inactivate pathogens localized within the protected tissues (Rajkowski and Thayer, 2001). Despite their effectiveness, these methods are difficult to implement commercially because of a narrow optimum range for the temperature or irradiation and exposure time (Buck et al., 2003).

Chemical treatments with chlorine, sodium hypochlorite, and calcium hypochlorite are currently used for seed sterilization (NACMCF, 1999). However, chlorine reacts with natural organic matter to form trihalomethanes in

\footnotetext{
*Comesponding author: changhoo@snu.ac.kr

※ Received 6 August 2014; Revised 23 August 2014; Accepted 5 September 2014. This study was supported by a Grant (Project no. 11106803-3-SB020) from Korea Institute of Planning and Evaluation for Technology of Food, Agriculture, Forestry and Fisheries (iPET).

(C) 2014 Korean Society for Horticultural Science
} 
water and trihalomethanes can cause cancer (Urano et al., 1983). Therefore, the need for the development of new sterilization technologies is increasing in order to reduce the accumulation of chemical residues and to maintain environmental and food safety (Richardson et al., 1998).

Ozone is a potential alternative for food sterilization due to its effectiveness in killing microorganisms and it offers a number of advantages over chemical treatment with chlorine compounds. The primary advantages in ozone treatment include its fast decomposition in water to oxygen, and the absence of residue or by-product (Wang et al., 2004). Researchers have previously tested the efficacy of ozonated water in the sterilization of alfalfa seeds and sprouts (Sharma et al., 2002; Singh et al., 2003; Wang et al., 2004).

Ozone has a high disinfectant activity, but it is not yet widely used due to its low solubility in water. Air-bubbles generated by air pump are $2-3 \mathrm{~mm}$ in diameter and they go up quickly and burst at the water surface. Therefore, the solubility of gas in water is very low. However, microbubbles rise more slowly to the water surface and the interior gas is completely dissolved in water because the diameters of micro-bubbles are less than $50 \mu \mathrm{m}$ (Ikeura et al., 2011a; Takahashi et al., 2007a). Moreover, microbubbles have special properties such as free radical generation, self-pressurization, and negative charge. In recent times, the application of micro-bubbles in the field of food and agricultural sciences has attracted great attraction due to these properties (Ikeura et al., 2011b; Sumikura et al., 2007; Takahashi et al., 2007b).

There have been several studies on the use of ozone micro-bubbles for removing residual pesticides from vegetables and reusing wastewater (Ikeura et al., 2011a, 2011b; Sumikura et al., 2007), however, this technique is unexplored for seed sterilization. Therefore, this study was undertaken to determine the effect of ozone micro-bubbles on seed sterilization in alfalfa sprouts. The effect of ozone microbubbles on seed viability was also investigated to determine the feasibility of commercial application of ozone microbubbles as a disinfectant in the alfalfa sprout industry.

\section{Materials and Methods}

\section{Observations of Alfalfa Seed Appearance}

Alfalfa seeds (Medicago sativa L.) were fixed in FAA (35\% formaldehyde:acetic acid:ethanol, $10: 1: 1)$ at $4^{\circ} \mathrm{C}$ for 24 hours. They were fixed with $1 \%$ osmium tetroxide in $50 \mathrm{mM}$ sodium phosphate buffer $(\mathrm{pH} \mathrm{7})$ at $4^{\circ} \mathrm{C}$ for 24 hours and dehydrated through a series of ethanol dilutions
(30, 40, 50, 60, 70, 80, 90, 95, and 100\%; 20 min each). The samples were stored overnight in $100 \%$ ethanol and further dehydrated by soaking in 100\% ethanol and then in $98 \%$ isoamyl acetate for 15 minutes each. After the removal of isoamyl acetate, they were dried by critical point drying and observed using scanning electron microscopy (SEM).

Measuring the Concentrations of Ozone Dissolved in Ozone Water and Ozone Micro-bubble Water

Ozone water was generated using an air pump combined with an ozone generator (OG-030V, Ozone Engineering Co. Ltd., Incheon, Korea) into $20 \mathrm{~L}$ of tap water. Ozone microbubbles were generated in water using a micro-bubble generator (Korea EMB Technology Co., Ltd., Seoul, Korea) and an ozone generator (OG-030V, Ozone Engineering Co., Ltd., Incheon, Korea), simultaneously. We activated the generators for 5 minutes and measured the concentrations of dissolved ozone every 30 seconds in ozone water and ozone micro-bubble water.

\section{Seed Sterilization Treatments}

Five treatments were evaluated for their efficacy in sterilization of alfalfa seeds: tab water (TW), micro-bubble water (MBW), ozone water (OW), ozone micro-bubble water (OMBW), and chlorine (CL) (Table 1). MBW was generated using a micro-bubble generator (Korea EMB Technology Co., Ltd., Seoul, Korea). OW and OMBW were generated as described above. CL was prepared with diluted commercial chlorine bleach $(0.5 \%)$. Alfalfa seeds were then soaked in these solutions for 5 minutes, while non-washed seeds were used as a control.

\section{Microbiological Analysis}

After sterilization, the seeds were rinsed three times with sterile distilled water and ground using a sterile mortar and pestle. One $\mathrm{mL}$ of ground seeds was blended

Table 1. The seed sterilization treatments used in this study.

\begin{tabular}{lcc}
\hline Treatments & Sanitizer & Concentration \\
\hline Control & Non-washed & - \\
TW & Tap water & - \\
MBW & Micro-bubble water & - \\
OW & Ozone water & $3.5 \mathrm{ppm}$ \\
OMBW & Ozone micro-bubble water & $5.3 \mathrm{ppm}$ \\
CL & Chlorine water & $0.5 \%$ \\
\hline
\end{tabular}


in $9 \mathrm{~mL}$ of sterile distilled water for 30 seconds. Appropriate decimal dilutions in sterile distilled water were then plated for standard plate count, E. coli, and Salmonella spp. Standard plate count (SPC) was determined by plating on standard plate count agar, incubating at $36^{\circ} \mathrm{C}$ for 24 hours, and counting colony-forming units (CFU). For determination of E. coli and Salmonella spp., we used the media of Desoxycholate agar and Salmonella Shigella agar, respectively, and incubated the plates at $36^{\circ} \mathrm{C}$ for 48 hours.

\section{Seed Viability Analysis}

To investigate changes in vitality of alfalfa seeds after sterilization, the percentage of germination and fresh weight of alfalfa sprouts was also measured at 5 days after sowing. One gram of sterilized seeds was placed in a Petri dish and irrigated with $3 \mathrm{~mL}$ of distilled water every day. Petri dishes were put in the chamber setting at $23^{\circ} \mathrm{C}$. Alfalfa seeds were germinated under dark condition for 3 days and after germination, alfalfa sprouts were cultivated at PPF $30 \mu \mathrm{mol} \cdot \mathrm{m}^{-2} \cdot \mathrm{s}^{-1}$ for 2 days.

\section{Statistical Analysis}

The experimental data were analyzed using SAS statistical software (SAS Institute, Cary, NC, USA). Duncan's multiple range tests were performed to determine any significant difference $(p \leq 0.05)$ among the various treatments.

\section{Results and Discussion}

\section{Appearance of Alfalfa Seed}

Although the visible appearance of seeds is smooth, there were many cracks and crevices on the surface of alfalfa seed when observing with a microscopy (Fig. 1). Pathogens can be harbored in cracks or crevices on the surface or between the seed coat and cotyledon of seeds (Sharma et al., 2002). Charkowski et al. (2002) observed alfalfa seeds inoculated with Salmonella enterica using microscopy and reported that $S$. enterica colonized the broken edges of alfalfa seed coats during the first 24 hours of seed germination.

\section{The Concentrations of Ozone Dissolved in Ozone Water and Ozone Micro-bubble Water}

Fig. 2 shows the change in the concentrations of ozone dissolved in OW and OMBW. The maximum concentrations of dissolved ozone were $3.5 \mathrm{ppm}$ at 5 minutes after the start of ozone generation and $5.3 \mathrm{ppm}$ at 2 minutes after the start of ozone micro-bubble generation in OW and OMBW, respectively. In both solutions, the concentration of dissolved ozone decreased gradually with time after 10 minutes from starting the generator. Thus, the concentration of dissolved ozone was kept higher and the time to maintain the maximum concentration of dissolved ozone was longer in OMBW.
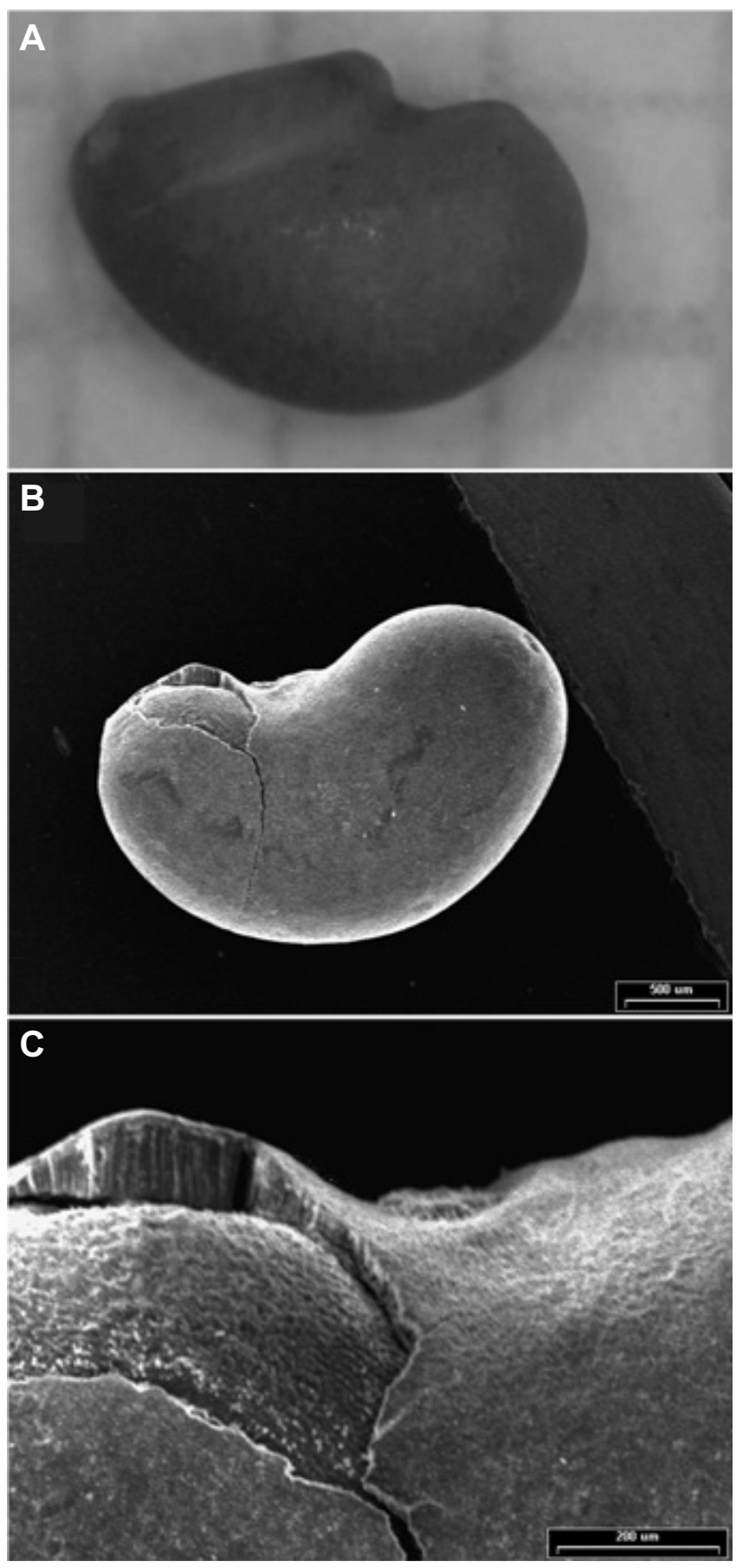

Fig. 1. The surface of alfalfa seed (A), scanning electron micrographs of alfalfa seed (B), and seed coat edge of alfalfa (C). 


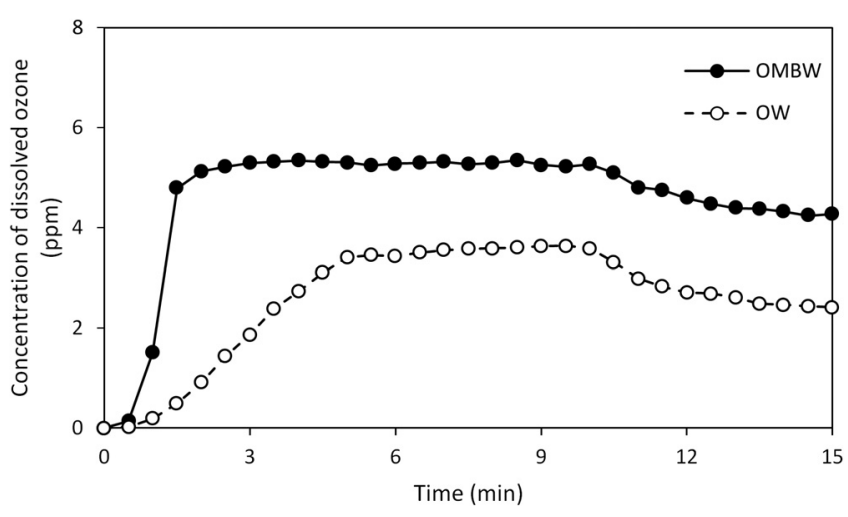

Fig. 2. Changes in the concentration of dissolved ozone after generating ozone for 5 minutes.

Table 2. The effect of sterilization treatments on total microbial population, E. coli and Salmonella spp. colonies in alfalfa seeds.

\begin{tabular}{lccc}
\hline Treatments & $\begin{array}{c}\text { Standard plate count } \\
\left(\log _{10} \text { CFU/mL) }\right.\end{array}$ & E. Coli & $\begin{array}{c}\text { Salmonella } \\
\text { spp. }\end{array}$ \\
\hline Control & $4.54 \mathrm{a}$ & Detected & N.D. \\
TW & $4.54 \mathrm{a}$ & Detected & N.D. \\
MBW & $4.17 \mathrm{~b}$ & Detected & N.D. \\
OW & $4.04 \mathrm{c}$ & N.D. & N.D. \\
OMBW & $1.88 \mathrm{~d}$ & N.D. & N.D. \\
CL & $1.75 \mathrm{e}$ & N.D. & N.D. \\
\hline
\end{tabular}

\section{Comparison of Aqueous Treatments for Sterilization and Viability of Alfalfa Seeds}

In non-washed alfalfa seeds (control), the SPC was approximately $4.54 \log _{10} \mathrm{CFU} / \mathrm{mL}$ and E. coli was detected (Table 2). There was no significant decrease in SPC between seeds washed with tap water and non-washed seeds. It indicated that washing with tap water did not affect the removal of pathogens from seeds. The treatments with MOBW and CL were most effective in reducing microbial populations, however, they did not result in complete eradication of pathogens. Salmonella spp. were not detected in all treatments and E. coli was successfully eliminated with OW, OMBW, and CL treatments. Other researchers (Sharma et al., 2002; Singh et al., 2003) have reported the effectiveness of ozone in seed sterilization and our study also shows that ozone can be used as an antimicrobial agent. However, the reduction of microbial populations by OW was significantly lower than that achieved with MOBW. The lower effectiveness of OW was probably due to the lower concentration of dissolved ozone and the inability to penetrate into damaged

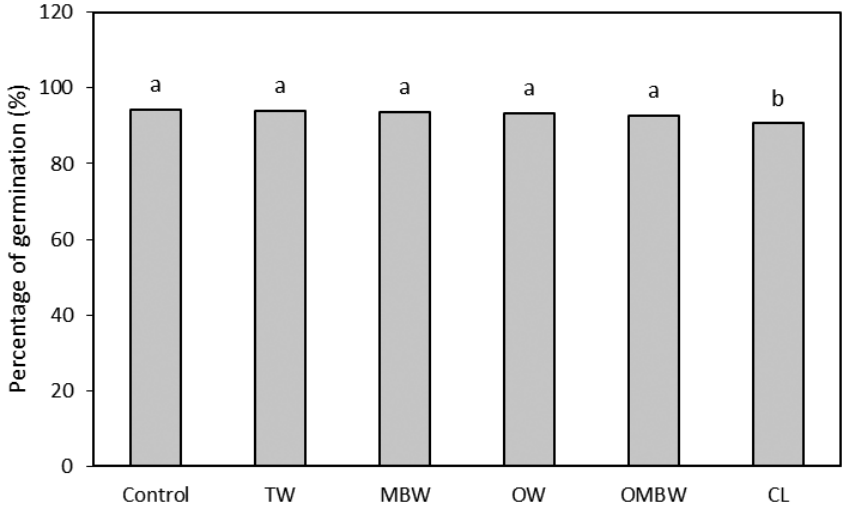

Fig. 3. The effect of sterilization treatments on the percentage of germination in alfalfa seeds. Means in columns followed by different letters are significantly different by Duncan's multiple range tests at $p \leq 0.05$.

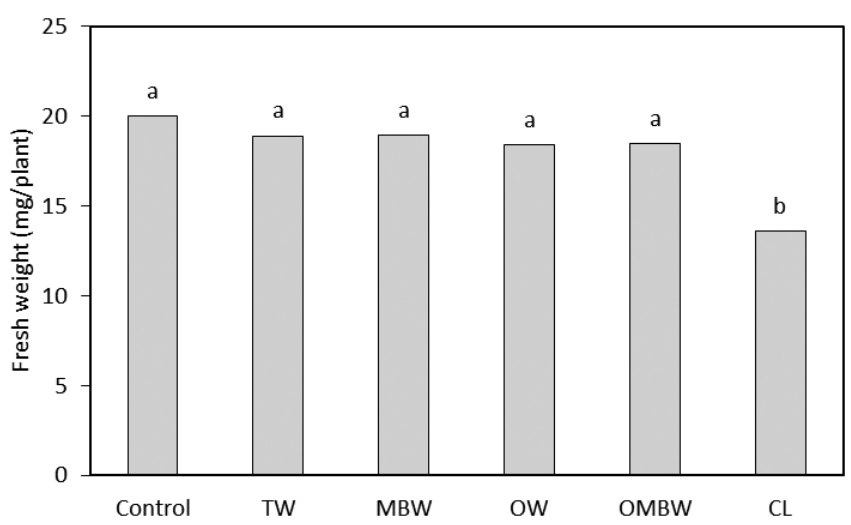

Fig. 4. The effect of seed sterilization treatments on fresh weight of alfalfa sprouts 5 days after sowing. Means in columns followed by different letters are significantly different by Duncan's multiple range tests at $p \leq 0.05$.

areas on the surface of alfalfa seeds. OMBW facilitated better penetration of ozone into the inaccessible cracks and crevices of alfalfa seeds, thus enhancing microbial decontamination.

Although the sterilization effect was highest with CL treatment, the percentage of germination and fresh weight of alfalfa sprouts after CL treatment were lower than those after the other treatments (Figs. 3 and 4). Successful seed sterilization must inactivate microbial pathogens while preserving seed viability (NACMCF, 1999). Beyond microbial consideration, the use of chlorine for sterilization of alfalfa seeds creates a problem with toxic residues and by-products and also requires numerous water rinses of the seeds after sterilization.

In conclusion, OMBW was the most efficient treatment 
for the sterilization and preservation of germination and growth potential of alfalfa seeds. The results of this study suggest that the application of OMBW as a disinfectant for seed sterilization in the production of alfalfa sprouts is feasible. However, pathogens could not be completely eliminated in this study. There is still a need to more thoroughly investigate the dissolved ozone concentration and soaking time of OMBW for complete sterilization of alfalfa seeds.

\section{Literature Cited}

Buck, J.W., R.R. Walcott, and L.R. Beuchat. 2003. Recent trends in microbiological safety of fruits and vegetables. Plant Health Prog. doi: 10.1094/PHP-2003-0121-01-RV.

Charkowski, A.O., J.D. Barak, C.Z. Sarreal, and R.E. Mandrell. 2002. Differences in growth of Salmonella enterica and Escherichia coli $\mathrm{O} 157: \mathrm{H} 7$ on alfalfa sprouts. Appl. Environ. Microbiol. 68:3114-3120.

Ikeura, H., F. Kobayashi, and M. Tamaki. 2011a. Removal of residual pesticide, fenitrothion, in vegetables by using ozone microbubbles generated by different methods. J. Food Eng. 103:345-349.

Ikeura, H., F. Kobayashi, and M. Tamaki. 2011b. Removal of residual pesticides in vegetables using ozone microbubbles. J. Hazardous Materials 186:956-959.

Michino, H., K. Araki, S. Minami, S. Takaya, N. Sakai, M. Miyazaki, A. Ono, and H. Yanagawa. 1999. Massive outbreak of Escherichia coli O157:H7 infection in schoolchildren in Sakai city, Japan, associated with consumption of white radish sprouts. Amer. J. Epidemiology 150:787-796.

National Advisory Committee on Microbiological Criteria for Foods (NACMCF). 1999. Microbiological safety evaluations and recommendations on sprouted seeds. Int. J. Food Microbiol. 52:123-153.

Rajkowski, K.T. and D.W. Thayer. 2001. Alfalfa seed germination and yield ratio and alfalfa sprout microbial keeping quality following irradiation of seeds and sprouts. J. Food Protection 64:1988-1995

Richardson, S.D., A.D. Thruston Jr., T.V. Caughran, T.W. Collette, K.S. Patterson, and B.W. Lykins, Jr. 1998. Chemical by-products of chlorine and alternative disinfectants. Food Technol. 52:58-61.

Scouten, A.J. and L.R. Beuchat. 2002. Combined effects of chemical, heat and ultrasound treatments to kill Samonella and Escherichia coli O157:H7 on alfalfa seeds. J. Appl. Microbiol. 92:668-674.

Sharma, R.R. and A. Demirci. 2003. Inactivation of Escherichia coli $\mathrm{O} 157: \mathrm{H} 7$ on inoculated alfalfa seeds with pulsed ultraviolet light and response surface modeling. J. Food Sci. 68:1448-1453.

Sharma, R.R., A. Demirci, L.R. Beuchat, and W.F. Fett. 2002. Inactivation of Escherichia coli $\mathrm{O} 157: \mathrm{H} 7$ on inoculated alfalfa seeds with ozonated water and heat treatment. J. Food Protection 65:447-451.

Singh, N., R.K. Singh, and A.K. Bhunia. 2003. Sequential disinfection of Escherichia coli O157:H7 inoculated alfalfa seeds before and during sprouting using aqueous chlorine dioxide, ozonated water, and thyme essential oil. LWT-Food Sci. Technol. 36: 235-243.

Sumikura, M., M. Hidaka, H. Murakami, Y. Nobutomo, and T. Murakami. 2007. Ozone micro-bubble disinfection method for wastewater reuse system. Water Sci. Technol. 56:53-61.

Takahashi, M., K. Chiba, and P. Li. 2007a. Free-radical generation from collapsing microbubbles in the absence of a dynamic stimulus. J. Phys. Chem. B 111:1343-1347.

Takahashi, M., K. Chiba, and P. Li. 2007b. Formation of hydroxyl radicals by collapsing ozone microbubbles under strongly acidic conditions. J. Phys. Chem. B 111:11443-11446.

Urano, K., H. Wada, and T. Takemasa. 1983. Empirical rate equation for trihalomethane formation with chlorination of humic substrates in water. Water Res. 17:1797-1801.

Wang, H., H. Feng, and Y. Luo. 2004. Microbial reduction and storage quality of fresh-cut cilantro washed with acidic electrolyzed water and aqueous ozone. Food Res. Intl. 37:949-956. 